\title{
The Role of Endotoxin during Typhoid Fever and Tularemia in Man. III. Hyperreactivity to Endotoxin during Infection *
}

\author{
Sheldon E. Greisman, $†$ Richard B. Hornick, and Theodore E. Woodward \\ (From the Departments of Medicine and Physiology, University of Maryland School of \\ Medicine, Baltimore, $M d$.)
}

The intravenous administration of endotoxins prepared from gram-negative bacteria induces profound physiologic derangements in man (2). Nevertheless, their clinical significance during illnesses induced by gram-negative bacteria remains speculative. One major objection to ascribing any significant role to endotoxin in mediating the pathophysiologic changes during gram-negative bacterial infections is based on the phenomenon of acquired tolerance. Thus, when endotoxins are administered daily to normal man as single intravenous injections, resistance to their toxic and pyrogenic activities develops rapidly (3). Such acquisition of tolerance has led some investigators to suggest that circulating endotoxin can play no major role in those infections in which fever is sustained $(4,5)$. Others have proposed that endotoxin can play an important role in sustained infection, e.g., typhoid fever, and that tolerance constitutes an important mechanism in recovery from the illness (6). Support for either of these concepts is obtained from the observation that a high degree of tolerance to the pyrogenic action of Salmonella typhosa and other bacterial endotoxins is observed during convalescence from typhoid fever and tularemia $(6,7)$. Nevertheless, inferences as to the importance of bacterial endotoxin during infection based upon observations of

* Submitted for publication March 9, 1964; accepted May 12, 1964.

Supported by the Commission on Epidemiological Survey of the Armed Forces Epidemiological Board, contract DA-49-007-MD-751, and U. S. Public Health Service research grant HE-07280-03.

A preliminary report of this work was presented at the Seventy-fifth Annual Meeting of the Association of American Physicians, May 1962 (1).

† Recipient of Career Development Award from the National Institutes of Health under contract HE-K3-15, 237-C3. endotoxin tolerance in normal or in convalescent man are not justified. Reactions to endotoxin must be studied in the infected host during the febrile phase of illness. The following studies were performed in man to define $a$ ) the effect of induced endotoxin tolerance on the subsequent febrile and clinical course of two gram-negative bacterial infections, typhoid fever and tularemia, and $b$ ) the influence of the typhoid and tularemic infections on the preinduced endotoxin tolerance.

\section{Materials and Methods}

Volunteers for these studies were male inmates of the Maryland House of Correction, Jessup, Md., whose ages ranged from 22 to 44 . Each volunteer was fully apprised of all aspects of the investigation and the attendant risk. They were free to cease participation at any time. Complete medical surveys were performed to verify the fitness of each participant.

Infectious agents and nature of clinical illness. The preparation and administration of the inocula of $S$. $t y$ phosa, Pasteurella tularensis, and sandfly fever virus and the clinical course and treatment of the resulting illnesses have been outlined previously (7). Convalescence is defined as beginning on the first day that the maximal oral temperature (as recorded at 12 and 8 a.m. and at 12,4 , and 8 p.m.) remained below $100^{\circ} \mathrm{F}$.

Bacterial endotoxins. Two preparations of bacterial endotoxins were employed: a highly purified endotoxin from $S$. typhosa (0-282), ${ }^{1}$ previously described (8), and Pseudomonas endotoxin 2 prepared by tryptic digestion (9). Both endotoxin preparations were suspended in sterile, pyrogen-free physiologic saline and stored in rubber-stoppered vaccine bottles at $4^{\circ} \mathrm{C}$. The $S$. typhosa endotoxin was diluted to a concentration of $0.25 \mu \mathrm{g}$ per $\mathrm{ml}$ and the Pseudomonas endotoxin to $10 \mu \mathrm{g}$ per $\mathrm{ml}$. The diluted endotoxin preparations were bacteriologically sterile, and the same bottle of diluted endotoxin was always employed throughout any given study. Po-

1 Kindly supplied by Dr. Maurice Landy, National Institutes of Health.

2 Kindly supplied as Piromen by Dr. T. A. Garrett, Baxter Laboratories, Morton Grove, Ill. 
tency of the diluted endotoxins was tested before the human studies by a standard pyrogen assay procedure employing acclimatized, loosely restrained, albino rabbits. Repeat assays conducted at the conclusion of the studies, in groups of five rabbits each with two dose levels for each group, indicated no loss of pyrogenicity.

Pyrogen assay in man. Volunteers were hospitalized, and all responses to endotoxin were assayed after the subjects were confined to bed and covered with a light blanket. Syringes were either of the sterile, pyrogenfree, disposable type ${ }^{3}$ or were heated overnight in a dry-

${ }^{3}$ Burron Medical Products, Bethlehem, $\mathrm{Pa}$. air oven to $180^{\circ} \mathrm{C}$ to eliminate extraneous pyrogens. Each test was begun between 8 and 9 a.m. Flexible thermocouples were inserted into the rectum for fixed distances ( 6 inches) and temperatures recorded by a telethermometer. 4 Temperatures were monitored immediately before and every half-hour after the intravenous injection of endotoxin. The results were plotted on standard graph paper, and the area under the 7-hour fever curve, with the initial temperature as the base line, was calculated by summing the number of enclosed small

4 Yellow Springs Instruments Co., Yellow Springs, Ohio.

TYPHOID STUDY A

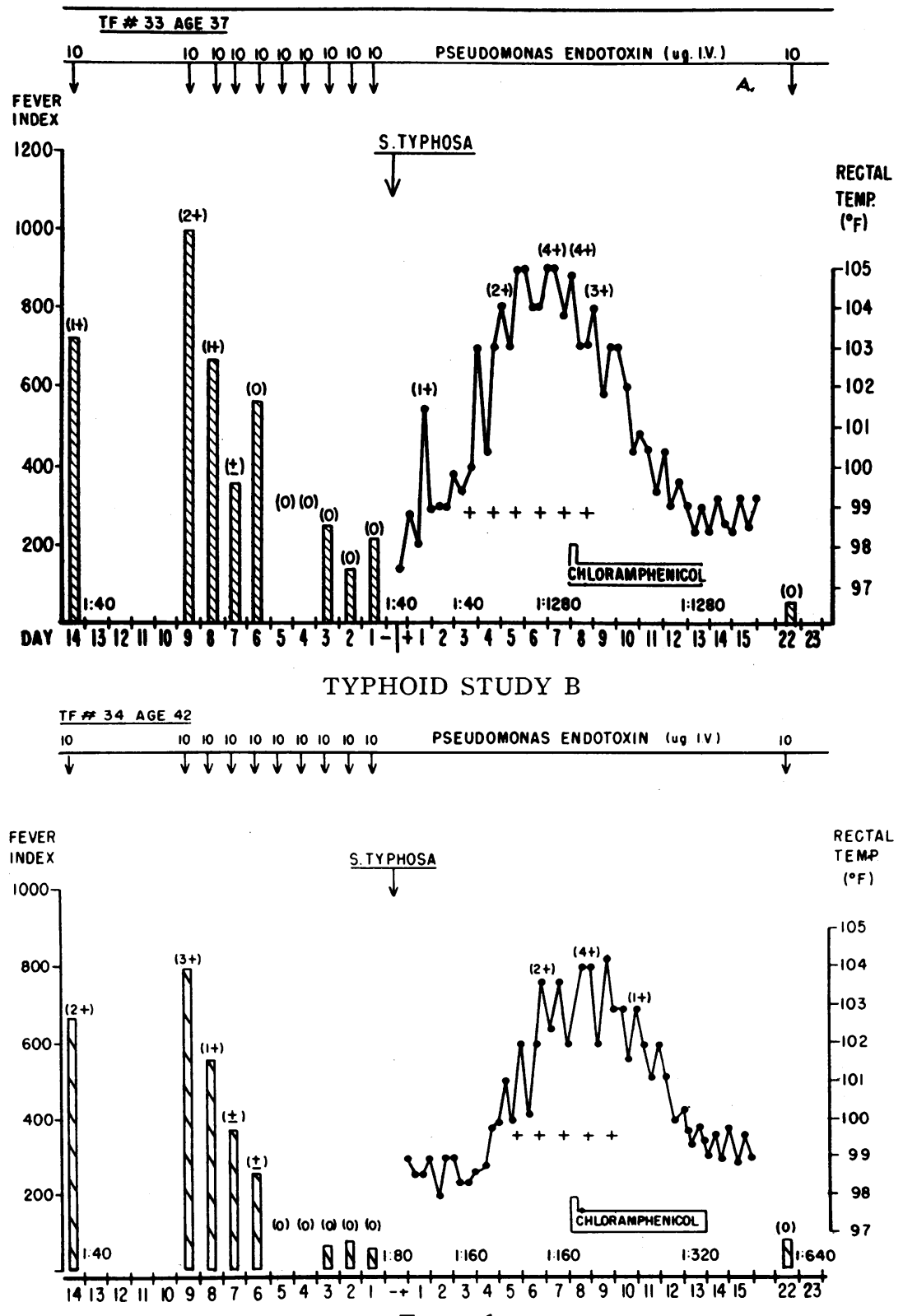

Figure 1. 


\section{TYPHOID STUDY C}

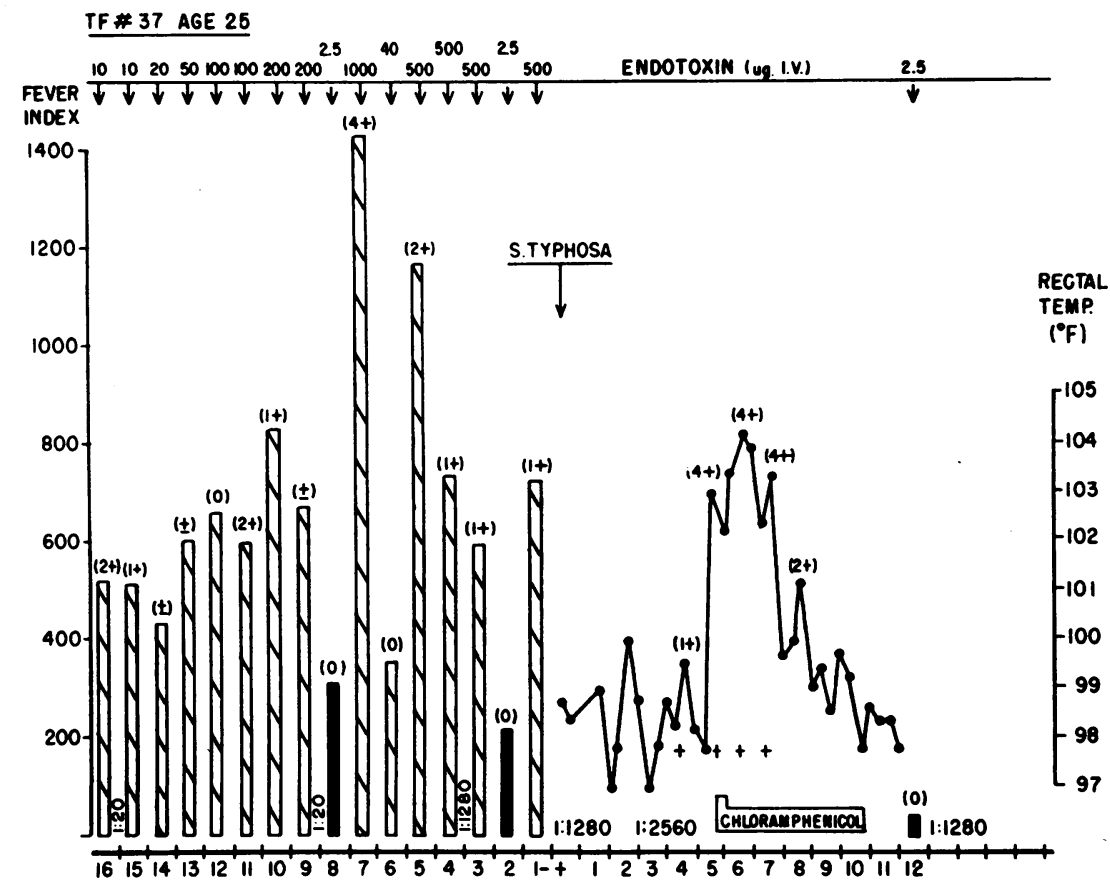

Fig. 1. TyphoId FeVER in endotoxin-tolerant subjects. A, B. Inability of PYROGENIC TOLERANCE INDUCED BY DAILY INTRAVENOUS INJECTIONS OF $10 \mu \mathrm{G}$ P seudomonas ENDOTOXIN TO MITIGATE TYPHOID FEVER. Note that tolerance is seen upon retesting on day 11 of afebrile convalescence. Figures in parentheses represent the intensity of subjective reactions (see text). S. typhosa $\mathrm{O}$ agglutinin titers are given above bottom line. $t=$ blood culture positive for $S$. typhosa. C. INABILITy of PYROGENIC TOLERANCE INDUCED BY DAILY INTRAVENOUS INJECTIONS OF INCREASING QUANTITIES OF Pseudomonas ENDOTOXIN TO INHIBIT TYPHOID FEVER. This subject was shown to possess cross-tolerance to $2.5 \mu \mathrm{g}$ of $S$. typhosa endotoxin on days 8 and 2 before challenge with viable $S$. typhosa (shaded bars). Note that tolerance to the $S$. typhosa endotoxin is demonstrable on day 4 of afebrile convalescence.

squares. This value, termed the fever index, represents a measure of the height and duration of the febrile responses (10). The ordinates were plotted so that 100 small squares represented a fever index of 100 and reflected a $1^{\circ} \mathrm{F}$ rise in rectal temperature for a 1-hour duration. The diurnal variation of normal body temperature was so great that temperatures seldom returned to preinjection values. In such instances, the fever curves were extrapolated to the base line. In addition to the fever index, subjective reactions (headache, chills, myalgia, anorexia) after endotoxin administration-were graded as follows: $1+=$ mild, $2+=$ moderate, $3+=$ severe, and $4+=$ extremely severe.

\section{Results}

Effect of endotoxin tolerance on induced typhoid fever. Before oral challenge with viable $S$. $t y$ phosa, two subjects were rendered tolerant by daily intravenous injections of $10 \mu \mathrm{g}$ Pseudomonas endotoxin (Figure $1 \mathrm{~A}$ and $\mathrm{B}$ ). A third volunteer was rendered tolerant by daily intravenous injections of progressively increasing quantities of the Pseudomonas endotoxin (Figure 1C). Pseudomonas, rather than $S$. typhosa endotoxin, was employed in these initial studies to circumvent the possible induction of specific immunity to the viable $S$. typhosa. In one subject, a high degree of cross-tolerance was created to $S$. typhosa endotoxin as evidenced by the minimal responses to slow infusions of $2.5 \mu \mathrm{g} S$. typhosa endotoxin administered on days 8 and 2 before challenge with viable $S$. typhosa organisms (Figure 1C). Five normal control subjects infused with onetenth this quantity of the same $S$. typhosa endotoxin preparation exhibited marked febrile and subjective reactions (mean fever index, 1,880; $\mathrm{SE} \pm 230$; mean subjective reaction, $3+$ ). Despite this tolerance to Pseudomonas and to $S$. 
TYPHOID STUDY A

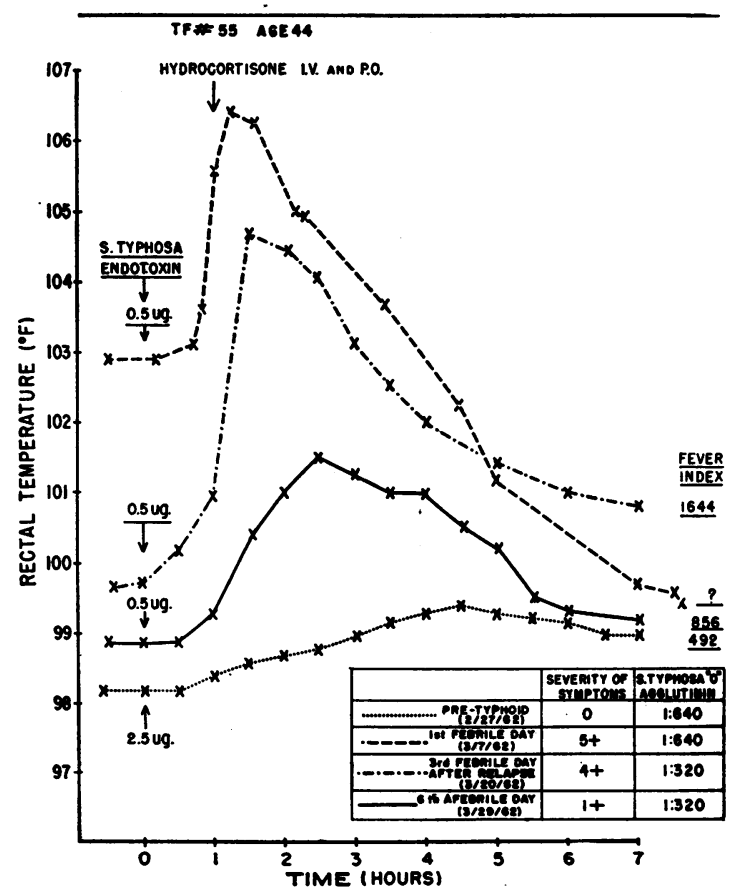

TYPHOID STUDY B

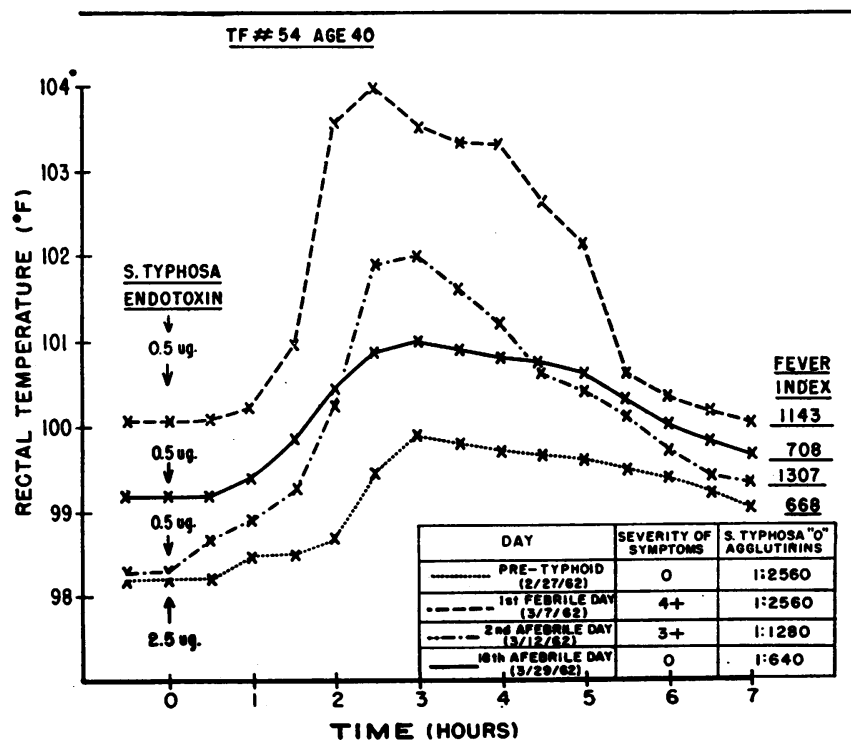

Fig. 2. A, B. Endotoxin Reactivity dURING TYPHoId FEVER. The subjects were initially rendered tolerant by daily intravenous injections of increasing quantities of $S$. typhosa endotoxin. The tolerant reaction to $2.5 \mu \mathrm{g} S$. typhosa endotoxin on day 21 is depicted by the lower curves. The subsequent hyperreactivity during typhoid illness and decline of such reactivity during convalescence are shown. 
typhosa endotoxin, the subsequent clinical course of illness after challenge with viable $S$. typhosa, including the pyrexia, toxemia, and bacteremia, was not mitigated, nor was the incubation period prolonged when compared with a nontolerant control group infected at the same time. Tolerance to the Pseudomonas and the $S$. typhosa endotoxins was again evident upon retesting after the third day of convalescence (Figure $1 \mathrm{~A}$ to $\mathrm{C}$ ).

Effect of typhoid fever on preinduced endotoxin tolerance. The observations that unmitigated typhoid illness develops in man despite preinduced tolerance to bacterial endotoxins sufficient to protect against febrile and toxic manifestations as severe as those seen during typhoid fever suggested initially that circulating endotoxin could play no major role in pathogenesis. Nevertheless, it remained to be demonstrated that the tolerance to endotoxin, although existing before and after illness, was maintained during illness. Two volunteers were given daily intravenous injections of $S$. typhosa endotoxin in increasing quantities over a 3-week period. The lowest curve in Figure $2 \mathrm{~A}$ illustrates the subsequent ability of the first subject to tolerate the intravenous administration of $2.5 \mu \mathrm{g} S$. typhosa endotoxin. The febrile response after this large dose of endotoxin was minimal, and no subjective discomfort was evident. Viable $S$. typhosa was now administered orally. Despite the elevated $S$. typhosa O agglutinin titer, overt typhoid illness developed as in the nontolerant control group. On the first day of illness, when the rectal temperature was $102.9^{\circ}$ $\mathrm{F}, 0.5 \mu \mathrm{g} S$. typhosa endotoxin was given intravenously. The subsequent febrile and subjective responses were so severe (upper curve, Figure $2 \mathrm{~A}$ ) that $100 \mathrm{mg}$ hydrocortisone was administered intravenously and $100 \mathrm{mg}$ orally to control the reactions. The subject was subsequently treated with chloramphenicol; after becoming af ebrile for several days, relapse ensued when medication was discontinued. On the third day of relapse, at a time when the rectal temperature was $99.7^{\circ} \mathrm{F}, 0.5$ $\mu \mathrm{g}$ of $S$. typhosa endotoxin was again administered intravenously; the resulting febrile and subjective reactions were again intense (second from top curve, Figure 2A). After a second course of antibiotic therapy, and after remaining afebrile for 6 days, $0.5 \mu \mathrm{g}$ of $S$. typhosa endotoxin given intravenously now induced only a mild febrile response and a minimal subjective reaction (second from bottom curve, Figure 2A). A similar reaction pattern to $S$. typhosa endotoxin was observed in the second volunteer (Figure $2 \mathrm{~B}$ ). This latter subject, although tolerant to $2.5 \mu \mathrm{g}$ of $S$. typhosa endotoxin given intravenously before typhoid illness and possessing a high $S$. typhosa $\mathrm{O}$ agglutinin titer, developed unmitigated typhoid illness after oral administration of viable $S$. typhosa. Hyperreactivity occurred to $0.5 \mu \mathrm{g}$ of the endotoxin given intravenously on the first day of illness, as well as early in convalescence ( 36 hours after becoming afebrile), but a tolerant reaction was again evident later in convalescence.

To define more precisely the time at which hyperreactivity to endotoxin appears and subsides during typhoid fever, and to determine the specificity of such hyperreactivity, four additional subjects were rendered tolerant to Pseudomonas endotoxin by daily intravenous injections of 25

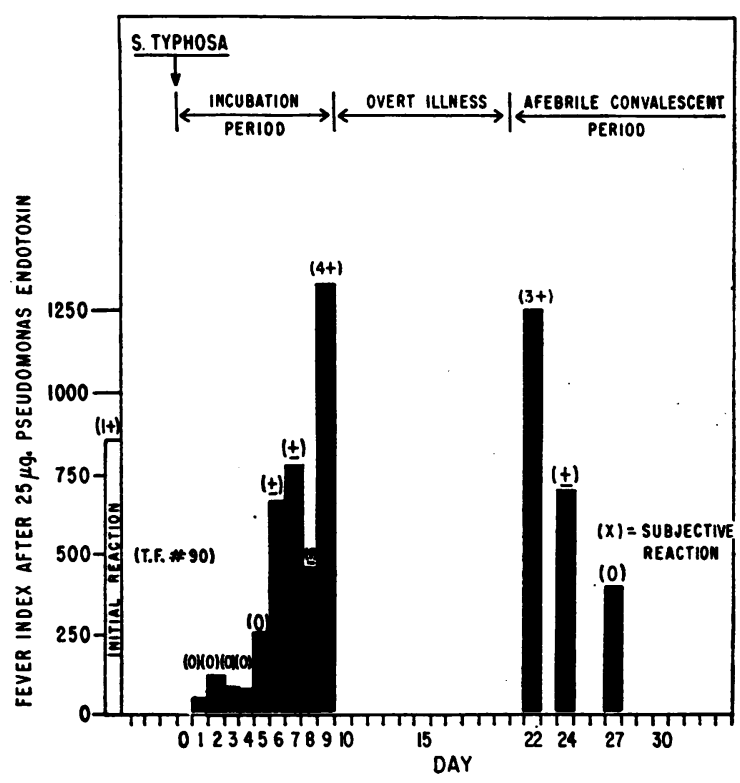

Fig. 3. Endotoxin REACTIVITY DURING TYPHOID FEVER. Typical reaction pattern depicting the stepwise development of hyperreactivity to $25 \mu \mathrm{g}$ Pseudomonas endotoxin in a tolerant subject during the incubation phase of typhoid fever and the stepwise decline of hyperreactivity during convalescence. Tolerance was induced before challenge with viable $S$. typhosa by daily intravenous injections of Pseudomonas endotoxin increasing from 25 to $250 \mu \mathrm{g}$ over a 30 -day period. The initial control reaction to $25 \mu \mathrm{g}$ of the endotoxin is shown by the unshaded bar on the left. 


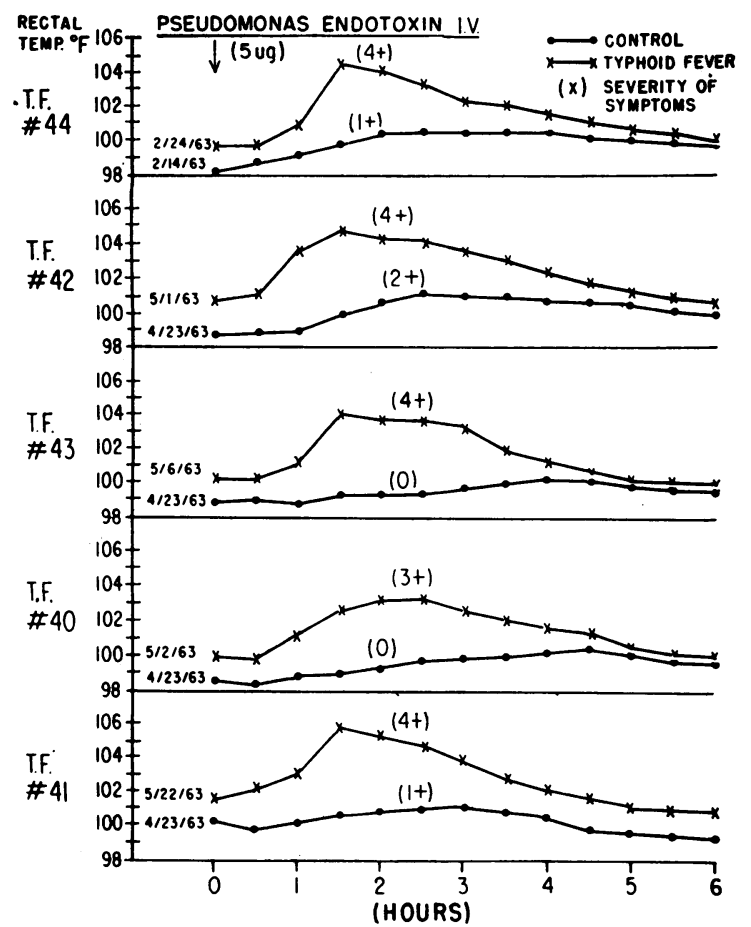

Fig. 4. Endotoxin ReACtivity DURING TYPhOId FEVER. Hyperreactivity to $5 \mu \mathrm{g}$ Pseudomonas endotoxin during typhoid fever is depicted. A single control fever index was obtained, and retesting was performed on the initial day of overt typhoid illness.

$\mu \mathrm{g}$, increasing to $250 \mu \mathrm{g}$ over a period of 30 days. These subjects were then infected by oral challenge with viable $S$. typhosa. During the incubation period, daily intravenous testing was performed with $25 \mu \mathrm{g}$ Pseudomonas endotoxin. As previously, unmitigated illness developed after the same incubation period as in the control group. The subsequent results were consistent in all volunteers; a typical reaction pattern to endotoxin is illustrated in Figure 3. There was a stepwise increase in reactivity to endotoxin until the day before overt illness, when reactivity exceeded the initial pretolerant level. During convalescence reactivity decreased in stepwise fashion. In all subjects hyperreactivity was evident during the first 2 afebrile convalescent days, and thereafter tolerance developed rapidly.

To determine whether hyperreactivity to endotoxin also developed in subjects not previously rendered tolerant to endotoxin, five volunteers were infected orally with viable $S$. typhosa after a single base-line pyrogen assay was obtained to $5 \mu \mathrm{g}$ Pseudomonas endotoxin. Testing was re- peated on the initial day of clinically overt typhoid illness (evidenced by onset of either headache or a rise in base-line 8 a.m. rectal temperature of over $1^{\circ} \mathrm{F}$, or both). The results are shown in Figure 4. In each subject, hyperreactivity to the endotoxin appeared at the onset of illness.

Effect of tularemia on preinduced endotoxin tolerance. Five normal subjects were given a daily intravenous injection of $0.25 \mu \mathrm{g} S$. typhosa endotoxin for 27 days. All subjects were challenged by intradermal inoculation of $P$. tularensis on day 27 of tolerance. Two days after challenge with $P$. tularensis (24 hours before the onset of overt illness), a slight decrease in tolerance was apparent (Figure 5A and B). On the third day postchallenge with $P$. tularensis, clinical illness was evident. The intravenous injection of less than one-quarter the quantity of $S$. typhosa endotoxin to which tolerance had existed the previous day now evoked severe pyrogenic and subjective reactions (top curves, Figure 5A and $\mathrm{B}$ ). Hyperreactivity to the endotoxin was also manifest on the first afebrile convalescent day; by the fourth afebrile day, tolerance had virtually fully returned (Figure 5A and B). As with typhoid fever, there was no evidence that the preinduced tolerance to endotoxin prolonged the incubation period or mitigated the clinical course of the tularemic illness.

Effect of sandfly fever on preinduced endotoxin tolerance. As controls for the typhoid fever and tularemia studies, endotoxin responsiveness was assayed during the course of sandfly fever. Four volunteers were rendered tolerant by a daily intravenous injection of $0.25 \mu \mathrm{g} S$. typhosa endotoxin for 8 days. These subjects were then infected with the virus of sandfly fever, and their reactivity to endotoxin was monitored during illness and early convalescence. Typical reaction patterns are shown in Figure $6 \mathrm{~A}$ and $\mathrm{B}$. In contrast to typhoid fever and tularemia, no subject exhibited increased reactivity to endotoxin during illness, despite the presence of fever, nor during the initial afebrile convalescent day. Two additional subjects were studied who were not rendered tolerant to endotoxin. Testing during the control period with a single intravenous injection of $10 \mu \mathrm{g}$ Pseudomonas endotoxin yielded base-line fever indexes of 1,274 and 573 ; retesting on the initial afebrile convalescent day of 
TULAREMIA STUDY A

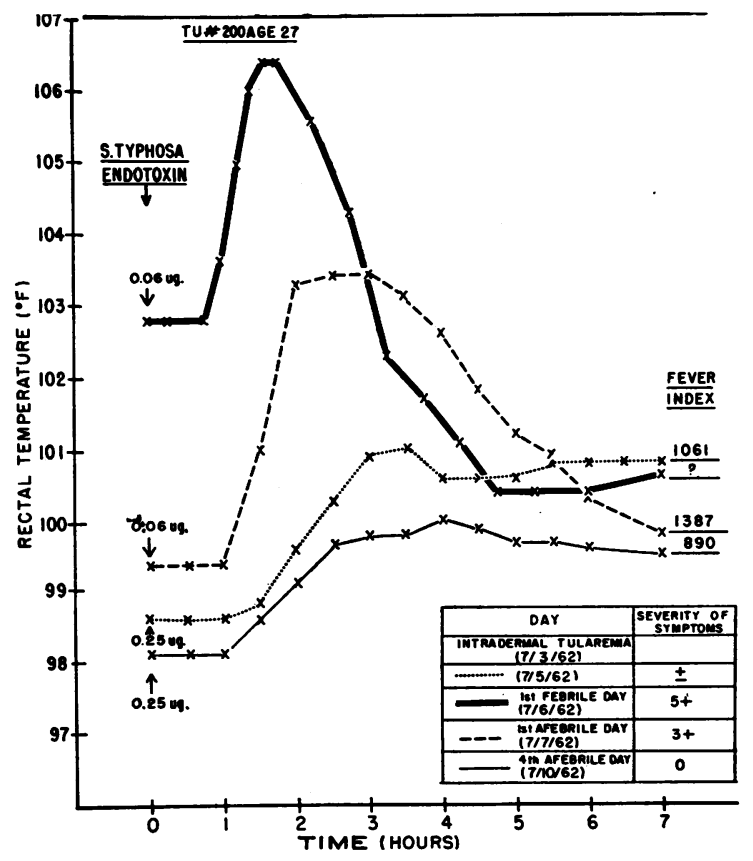

TULAREMIA STUDY B

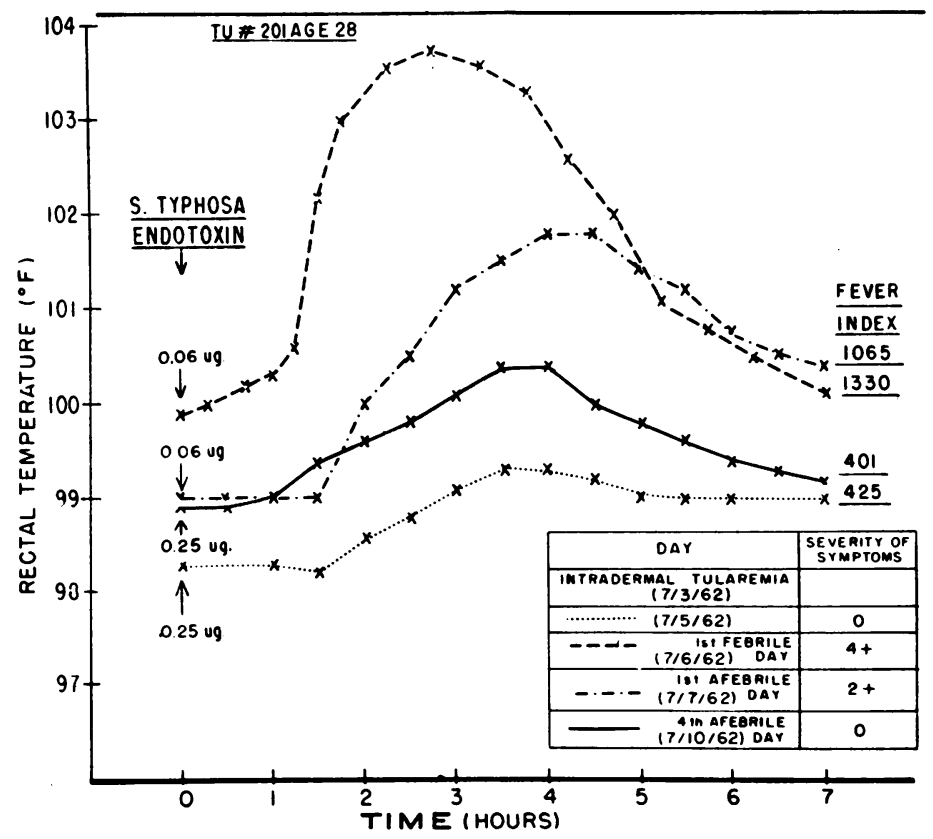

Fig. 5. A, B. Endotoxin Reactivity during tularemia. Typical effect of tularemia on preinduced tolerance to $S$. typhosa endotoxin. Tolerance was induced by a daily intravenous injection of $0.25 \mu \mathrm{g}$ $S$. typhosa endotoxin for 27 days. The fever indexes on day 27 $(7 / 3 / 62)$ were 717 and 400 for subjects TU no. 200 and TU no. 201 (A and $B$ ), respectively. Note the hyperreactivity during illness and the initial afebrile convalescent day (upper two curves), and the decline of reactivity by the fourth afebrile day. 


\section{SANDFLY FEVER STUDY A}

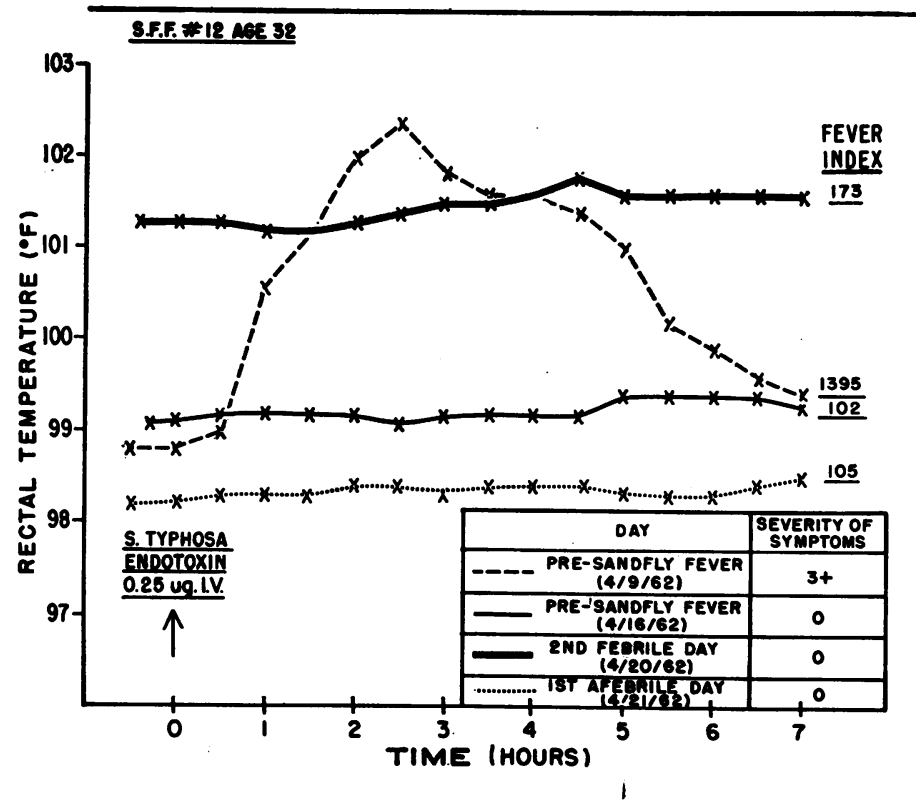

SANDFLY FEVER STUDY B

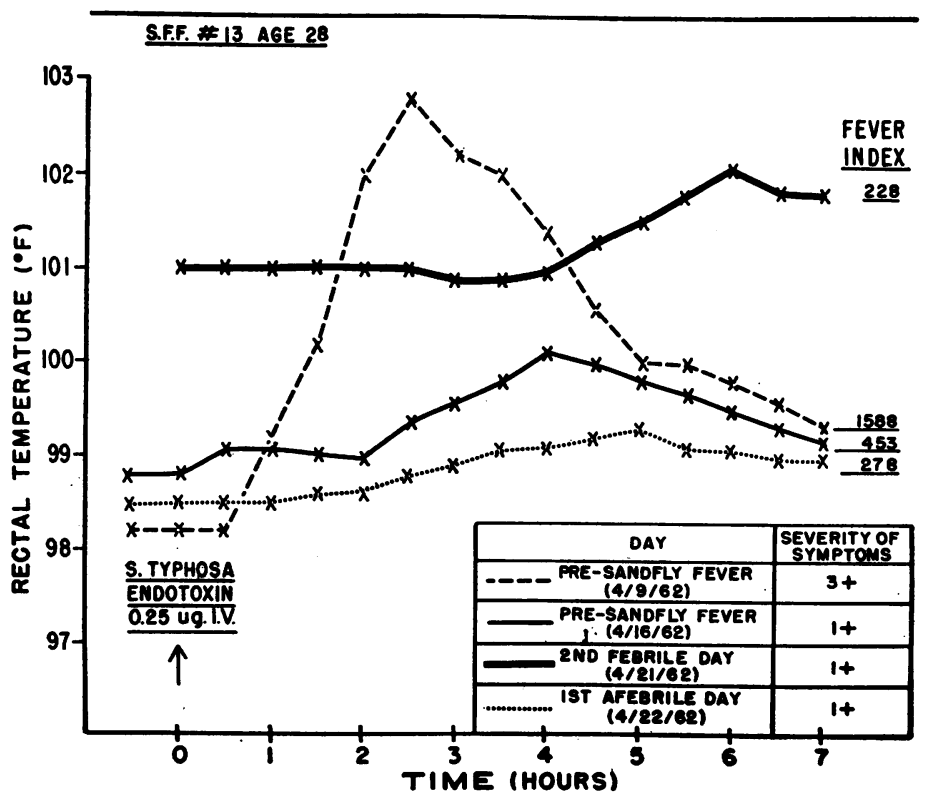

Fig. 6. A, B. ENDotoxin REACTIVITY DURING INFECTION WITH SANDFLY FEVER VIRUS. Typical reaction patterns depicting the lack of effect of sandfly fever infection on preinduced tolerance to $0.25 \mu \mathrm{g}$ $S$. typhosa endotoxin. The initial control reaction and the tolerant reaction to the $S$. typhosa endotoxin after a daily intravenous injection for 8 days are shown, as well as the subsequent responses on the second febrile day of sandfly fever and on the first afebrile convalescent day. 
sandfly fever 1 week later yielded fever indexes of 1,013 and 460 , respectively. Such tolerance is consistent with that expected after a single intravenous test dose of the endotoxin 1 week previously (7).

\section{Discussion}

Hyperreactivity to bacterial endotoxins, employing lethality as a criterion, occurs during a variety of infectious illnesses induced in laboratory animals $(11,12)$. In evaluating possible mechanisms for such hyperreactivity, the parameter for judging hyperreactivity to endotoxin during infection must be carefully considered. Thus, although induced tolerance to bacterial endotoxins is accompanied by resistance to most of its toxic activities (13), the converse cannot be assumed, i.e., that hyperreactivity to endotoxin, measured by lethality, indicates that its other toxic activities are enhanced. Indeed, it has been demonstrated that the enhanced susceptibility to the lethal activity of endotoxin seen in laboratory animals during infection with Mycobacterium tuberculosis is not accompanied by enhanced pyrogenic responses $(14,15)$. These findings indicate that generalized changes in metabolic activity of the host during active infection need not per se augment pyrogenic responsiveness; such observations are important in the analysis of augmented pyrogenic responses to endotoxin during infection in man.

Detailed data describing alterations in responsiveness to the pyrogenic activity of endotoxin during induced infection in laboratory animals with agents other than Myobacteria are not available. In man, increased reactivity to the pyrogenic activity of Brucella endotoxin has been established during active brucellosis (16). Specific hypersensitivity induced by the infection was suggested as the mechanism underlying such hyperreactivity to the Brucella endotoxin. Our present studies demonstrate that augmented pyrogenic responses to bacterial endotoxin also occur in man during two other infectious diseases, typhoid fever and tularemia. We emphasize that a real augmentation of pyrogenic responsiveness to endotoxin occurs during illness in that the increments in body temperature were consistently greater in both rate and magnitude, and usually duration, i.e., fever indexes were higher than those seen during the control period. This is in sharp distinction to observations by Bannister in man (17) and by Atwood and Kass in rabbits (18), wherein the injection of endotoxin during fever induced by exposure to a hot environment led to increments in body temperature (rate, magnitude, and duration) no greater than those seen under normal environmental conditions. Although body temperatures after endotoxin were higher in the hot environment, the initial temperatures were correspondingly elevated; these reactions represent additive rather than hyperreactive pyrogenic responses to endotoxin.

The unique opportunity to study the present volunteers before infection permitted observations bearing on the mechanism and significance of their hyperreactivity to endotoxin during infection: a) Since the role of acquired hypersensitivity in the production of hyperreactivity to endotoxin remains unproven, it is significant that augmented pyrogenic responses occurred to Pseudomonas endotoxin during typhoid fever and to $S$. typhosa endotoxin during tularemia. Certainly, therefore, the hyperreactivity cannot be attributed to hypersensitivity restricted to the specific endotoxin possessed by the infectious agents. Whether the hyperreactivity is more intense to endotoxins prepared from the homologous infecting organisms remains to be quantitated.

b) Augmented pyrogenic responsiveness to endotoxin appeared during the later portion of the incubation phase of typhoid illness and persisted during early convalescence, occurring before and after overt clinical illness. It cannot be attributed, therefore, simply to a summation of toxic effects in the overtly ill host. Moreover, since the enhanced pyrogenic responses occurred in the $a b-$ sence of elevations of rectal temperature, resetting of the thermoregulatory mechanisms appears unlikely as an explanation. The unaltered pyrogenic responses to endotoxin in control subjects infected with the virus of sandfly fever provide additional evidence to exclude nonspecific effects of infection or fever per se. The absence of augmented pyrogenic reactivity to endotoxin during fever induced by a hot environment, as mentioned previously $(17,18)$, is also consistent with this thesis.

c) Augmented pyrogenic responses occurred regardless of whether a high degree of tolerance 
to the endotoxin was induced before infection by repeated daily intravenous injections or whether only a single control intravenous base-line test was performed. It is not yet certain, however, whether the hyperreactivity involves the inhibition of those mechanisms that participate in tolerance or whether the tolerance mechanisms remain functional and offer some partial protection, i.e., whether a significant difference in the degree of hyperreactivity to endotoxin exists between those subjects with and without preinduced tolerance.

d) The hyperreactivity to bacterial endotoxin during typhoid fever and tularemia, in contrast to brucellosis (16), wanes rapidly once convalescence begins. This temporal course of the subsidence of the hyperreactivity to endotoxin reconciles an apparent conflict in earlier reports. Heyman and Beeson (19) observed hyperreactivity to the pyrogenic action of endotoxin during convalescence from typhoid fever and tularemia, whereas Neva and Morgan (6) and subsequently our own group reported tolerance ( 7$)$. It is important that the former investigators tested for tolerance on the second to third afebrile convalescent days, whereas the latter investigators tested after the third afebrile day. It is during this early convalescent period that the transition from hyper- to hyporeactivity is in progress.

$e)$ Endotoxin tolerance has been suggested to play an important role in recovery from gramnegative bacterial infections such as typhoid fever (6). This thesis appears untenable, since 1) induction of endotoxin tolerance sufficient to protect against febrile and toxic manifestations as severe as those seen during overt infection failed to prolong the incubation phase or mitigate subsequent typhoid or tularemic illness, 2) tolerance did not develop during overt illness, 3) recovery from typhoid fever, after initiation of chloramphenicol therapy, commenced while hyperreactivity to endotoxin was still apparent, and 4) recovery from typhoid fever, before the advent of chloramphenicol therapy, has been reported while hyperreactivity to endotoxin was still evident (19).

$f$ ) It has been suggested that since tolerance to endotoxin develops readily in normal subjects, circulating endotoxin can play no major role in those infections in which fever is sustained $(4,5)$. This argument against implicating endotoxin, at least with regard to the pathogenesis of typhoid fever and tularemia, is no longer tenable. On the contrary, the present findings show that the host remains capable of hyperreacting to minute quantities of endotoxin that may be released from the infecting microbes. Such findings, in conjunction with prior evidence for the release of physiologically active quantities of endotoxin during typhoid fever $(7,20)$, suggest an important role for circulating endotoxin in the pathogenesis of this illness.

Studies in man are currently in progress to define the mechanisms responsible for the augmented pyrogenic reactions to bacterial endotoxins during typhoid fever and tularemia.

\section{Summary}

The present studies evaluate the importance of bacterial endotoxin in the pathogenesis of typhoid fever and tularemia in man. Induction of endotoxin tolerance sufficient to protect against febrile and subjective toxic manifestations as severe as those seen during overt infection failed to prolong the incubation period or mitigate the subsequent clinical course of induced typhoid fever or tularemia. Although such findings appeared to militate against the importance of endotoxin in the pathogenesis of these illnesses, testing during overt infection revealed profound alterations in reactivity to endotoxin characterized by markedly augmented pyrogenic and subjective responses. This hyperreactivity to endotoxin occurred regardless of whether a high degree of tolerance was induced before infection by repeated daily intravenous injections of endotoxin or whether only a single injection was given intravenously to establish a base-line response. The hyperreactivity was not restricted to the endotoxin prepared from the infectious agent; increased reactivity was seen to Pseudomonas endotoxin during typhoid fever and to $S$. typhosa endotoxin during tularemia. Temporal studies after ingestion of viable $S$. typhosa indicated a stepwise onset of hyperreactivity to Pseudomonas endotoxin during the incubation period, with hyperreactivity consistently present 24 hours before overt illness, and stepwise subsidence of reactivity during convalescence, with tolerance after the third afebrile day. Control studies in febrile subjects infected with the virus 
of sandfly fever revealed no alteration in preinduced tolerance to $S$. typhosa endotoxin.

The present studies refute the concept that endotoxin tolerance plays an important role in recovery from typhoid fever or that the ability of normal man to develop tolerance to endotoxin precludes the importance of circulating endotoxin in the pathogenesis of sustained infectious illnesses. The mechanisms for the hyperreactivity to the pyrogenic activity of endotoxin during typhoid fever and tularemia in man are unknown and are currently under study.

\section{Acknowledgments}

The authors express their deepest appreciation to the volunteers who contributed so faithfully to these studies; to Mr. Paul Sharpley, who assisted in the care of these patients; to Mr. James W. Curran, Commissioner of the Maryland Department of Correction; to Mr. William F. Steiner, Chief Warden of the Maryland House of Correction; and to the other officials of the Maryland House of Correction for their generous cooperation and interest, without which these studies could not have been accomplished.

\section{References}

1. Greisman, S. E., R. B. Hornick, F. A. Carozza, Jr., and T. E. Woodward. Effect of endotoxin tolerance on human typhoid fever. Trans. Ass. Amer. Phycns 1962, 75, 170.

2. Favorite, G. O., and H. R. Morgan. Effects produced by the intravenous injection in man of a toxic antigenic material derived from Eberthella typhosa: clinical, hematological, chemical, and serological studies. J. clin. Invest. 1942, 21, 589.

3. Morgan, H. R. Resistance to the action of the endotoxins of enteric bacilli in man. J. clin. Invest. 1948, 27, 706.

4. Beeson, P. B. Discussion of W. W. Spink, The significance of endotoxin in brucellosis: experimental and clinical studies. Trans. Ass. Amer. Phycns 1954, 67, 292.

5. Atkins, E. Pathogenesis of fever. Physiol. Rev. 1960, 40, 580.

6. Neva, F. A., and H. R. Morgan. Tolerance to the action of endotoxins of enteric bacilli in patients convalescent from typhoid and paratyphoid fevers. J. Lab. clin. Med. 1950, 35, 911.
7. Greisman, S. E., R. B. Hornick, F. A. Carozza, Jr., and T. E. Woodward. The role of endotoxin during typhoid fever and tularemia in man. I. Acquisition of tolerance to endotoxin. J. clin. Invest. 1963, 42, 1064.

8. Webster, M. E., J. F. Sagin, M. Landy, and A. G. Johnson. Studies on the $\mathrm{O}$ antigen of Salmonella typhosa. I. Purification of the antigen. J. Immunol. 1955, 74, 455.

9. Nesset, N. M., J. McLallen, P. Z. Anthony, and L. G. Ginger. Bacterial pyrogens. I. Pyrogenic preparation from a Pseudomonas species. J. Amer. pharm. Ass., sci. Ed. 1950, 39, 456.

10. Beeson, P. B. Tolerance to bacterial pyrogens. I. Factors influencing its development. J. exp. Med. 1947, 86, 29.

11. Abernathy, R. S., G. M. Bradley, and W. W. Spink. Increased susceptibility of mice with brucellosis to bacterial endotoxins. J. Immunol. 1958, 81, 271.

12. Suter, E., and E. M. Kirsanow. Hyperreactivity to endotoxin in mice infected with Mycobacteria. Induction and elicitation of the reactions. Immunology 1961, 4, 354.

13. Thomas, L. The physiological disturbances produced by endotoxins. Ann. Rev. Physiol. 1954, $16,467$.

14. Hall, C. H., Jr., and E. Atkins. Studies on tuberculin fever. I. The mechanism of fever in tuberculin hypersensitivity. J. exp. Med. 1959, 109, 339.

15. Suter, E. Hyperreactivity to endotoxin after infection with BCG. Studies on its distinguishing properties. J. Immunol. 1964, 92, 49.

16. Abernathy, R. S., and W. W. Spink. Studies with Brucella endotoxin in humans: the significance of susceptibility to endotoxin in the pathogenesis of brucellosis. J. clin. Invest. 1958, 37, 219.

17. Bannister, R. G. Anhidrosis following intravenous bacterial pyrogen. Lancet 1960, 2, 118.

18. Atwood, R. P., and E. H. Kass. Relationship of body temperature to the lethal action of bacterial endotoxin. J. clin. Invest. 1964, 43, 151.

19. Heyman, A., and P. B. Beeson. Influence of various disease states upon the febrile response to intravenous injection of typhoid bacterial pyrogen. J. Lab. clin. Med. 1949, 34, 1400.

20. Greisman, S. E., R. B. Hornick, F. A. Carozza, Jr., and T. E. Woodward. The role of endotoxin during typhoid fever and tularemia in man. II. Altered cardiovascular responses to catecholamines. J. clin. Invest. 1964, 43, 986. 